\title{
Recanalization of Acute and Subacute Femoropopliteal Artery Occlusions with the Rotarex Catheter: One Year Follow-up, Single Center Experience
}

\author{
Sylvain R. Duc, ${ }^{1}$ Eric Schoch, ${ }^{1}$ Markus Pfyffer, ${ }^{2}$ Regula Jenelten, ${ }^{2}$ \\ Christoph L. Zollikofer ${ }^{1}$ \\ ${ }^{1}$ Department of Radiology, Kantonsspital Winterthur, Brauerstrasse 15, 8401 Winterthur, Switzerland \\ ${ }^{2}$ Department of Internal Medicine, Section of Angiology, Kantonsspital Winterthur, Brauerstrasse 15, 8401 Winterthur, Switzerland
}

\begin{abstract}
Purpose: To assess the efficacy and safety of a new rotational catheter for percutaneous removal of fresh and organized thrombi in the femoropopliteal artery.

Methods: Forty-one limbs in 38 patients (age 56-90 years, mean 75.6 years) with acute, subacute or chronic femoropopliteal occlusions of 1-180 days' duration (mean 31.6 days) were treated with the Rotarex device. The Fontaine stage was mainly IIB (Rutherford 2-3, 22 patients) or III (Rutherford 4, 14 patients). The length of occlusion varied from 2 to $35 \mathrm{~cm}$ (mean $13.1 \mathrm{~cm}$ ). After recanalization percutaneous transluminal angioplasty (PTA) was performed if there was a residual stenosis of $>25 \%$. Patients were followed up with color Doppler ultrasound at $48 \mathrm{hr}$ and clinically with Doppler pressures and oscillometry at 3,6, and 12 months.

Results: After an average of two passages with the Rotarex catheter all but two limbs required PTA for residual stenosis $>25 \%$. Five patients needed additional stenting. Major complications were one groin hematoma requiring blood transfusion and one arteriovenous fistula spontaneously thrombosing after unsuccessful primary prolonged balloon dilation. Distal embolizations occurred in 10 patients; 6 clinically relevant emboli were aspirated. All occlusions were technically successfully recanalised there were 2 early reocclusions after 1 day and two at 2 weeks. Brachial-ankle indices improved from an average of 0.41 before to 0.93 after recanalization. Primary and secondary patency rates were $62 \% / 84 \%$ after 6 months and 39\%/68\% after 1
\end{abstract}

Correspondence to: S.R. Duc, M.D., Department of Radiology, Balgrist University Hospital, Forchstrasse 340, 8008 Zurich, Switzerland; email: Sylvain.duc@balgrist.ch year. The amputation-free survival at 12 months was $100 \%$. Conclusion: The Rotarex mechanical thrombectomy device is an efficient, quick, easy to handle, and safe tool for the treatment of acute, subacute or even chronic peripheral arterial thromboembolic occlusions. It can be used for short or long occlusions with equal success, provided the obstruction is not heavily calcified and has been safely passed with a guidewire first.

Key words: Arteries/Femoral—arteries/Thrombosis— thrombectomy

The treatment of acute and subacute thromboembolic and local thrombotic ischemic lesions of the lower extremities has undergone considerable changes over recent years. The standard modality of surgical thrombectomy with the Fogarty balloon catheter technique for acute arterial occlusions has been replaced by percutaneous catheter techniques at many institutions. The simplest technique is percutaneous aspiration thrombectomy (PAT) for thrombus aspiration [1, 2]. Alternatively catheter-directed pharmacologic thrombolytic therapy with or without additional catheter aspiration is used, particularly if the occlusion is already a few days or weeks old [3, 4]. These techniques obtain the best results in acute occlusions of less than 2 weeks' duration [1].

Various mechanical devices have been introduced which involve maceration or fragmentation and removal of thrombus. There are two categories of devices for mechanical thrombectomy (MT): (1) rotational recirculation devices which work by the vortex principle, such as the Amplatz thrombectomy catheter (ATD, Microvena, White Bear Lake, MN) or the Arrow-Trerotola PTD (Arrow International, Reading, PA); and (2) hydrodynamic (rheo- 
lytic) recirculation devices which operate on the principle of the Venturi effect, such as the Hydrolyser (Cordis, Johnson and Johnson, Miami, FL), Oasis (Boston Scientific, Galway, Ireland), and the Angiojet (RTC; Possis Medical, Minneapolis, MN) [5-10]. These devices are not suited for subacute occlusions of more than 7-14 days' duration. Recently a new rotational mechanical thrombectomy catheter, the Straub Rotarex (Straub Medical, 7323 Wangs, Switzerland) has been introduced in Europe. This device combines the two essential effects of mechanical clot fragmentation and removal of the fragmented clot material from the vessel by negative pressure $[11,12]$.

The purpose of the following retrospective report from a single center is to present the 1 year follow-up results after recanalization of acute and subacute femoropopliteal occlusions with the Rotarex catheter. To our knowledge this study includes the largest number of patients treated primarily with the Rotarex catheter for occlusions of the native femoropopliteal arterial axis with a 12 month follow-up including primary and secondary patency rate survival curves.

\section{Materials and Methods}

\section{The Rotarex Catheter and its Application}

The Rotarex catheter consists of a coated stainless steel spiral rotating inside an 8 Fr single-lumen tetrafluorethylene-hexafluorpropylene copolymerizate (FEP) catheter. The tip of the catheter consists of a dual-blade windowed cutting crown of which the inner blade or stator is connected to the catheter shaft. The outer blade or so-called rotor is connected to the rotating spiral which is driven by a $40 \mathrm{~W}$ reusable electric motor unit. The spiral and motor are connected before use by a magnetic clutch. The rotating spiral running at $40,000 \mathrm{rpm}$ creates a vacuum at the catheter tip and the abraded fresh or organized occlusion material is sucked through the cutting slits, fragmented, and transported by the rotating spiral through the catheter lumen into a collecting bag (Fig. 1).

Since the Rotarex is an over-the-wire system ( 0.020 inch) any occlusion needs first to be recanalized by conventional guidewire technique. After assessment of the lesion by direct antegrade angiography via a common femoral artery approach the occlusion is recanalized with a Terumo $\mathrm{J}$ wire (0.035 inch) (Terumo, Somerset, NJ) and a $4 \mathrm{Fr}$ angiographic catheter is placed across the occlusion to allow the exchange for an 0.018 inch guidewire (Boston Scientific). Afterwards, the device is introduced through an 8 Fr hemostatic sheath and the catheter is activated while its tip is still proximal to the occlusion to allow lubrication of the spiral inside the catheter with the aspirated blood. Then the catheter is slowly advanced into the occlusion in a to-and-fro motion with occasional retraction into the already recanalized lumen (to allow cooling of the catheter by the inflowing fresh blood) (Fig. 2). Care must be taken to achieve sufficient cooling of the catheter tip and evacuation of the debris to get an appropriate blood flow along the catheter. In order to minimize peripheral embolization of clot the distal end of the occlusion should not be passed too fast before all loose material has been sucked back into the catheter. The device allows reopening of approximately $10 \mathrm{~cm}$ of occlusion within 30 $60 \mathrm{sec}$. Several passages of the occlusion may be needed to clean

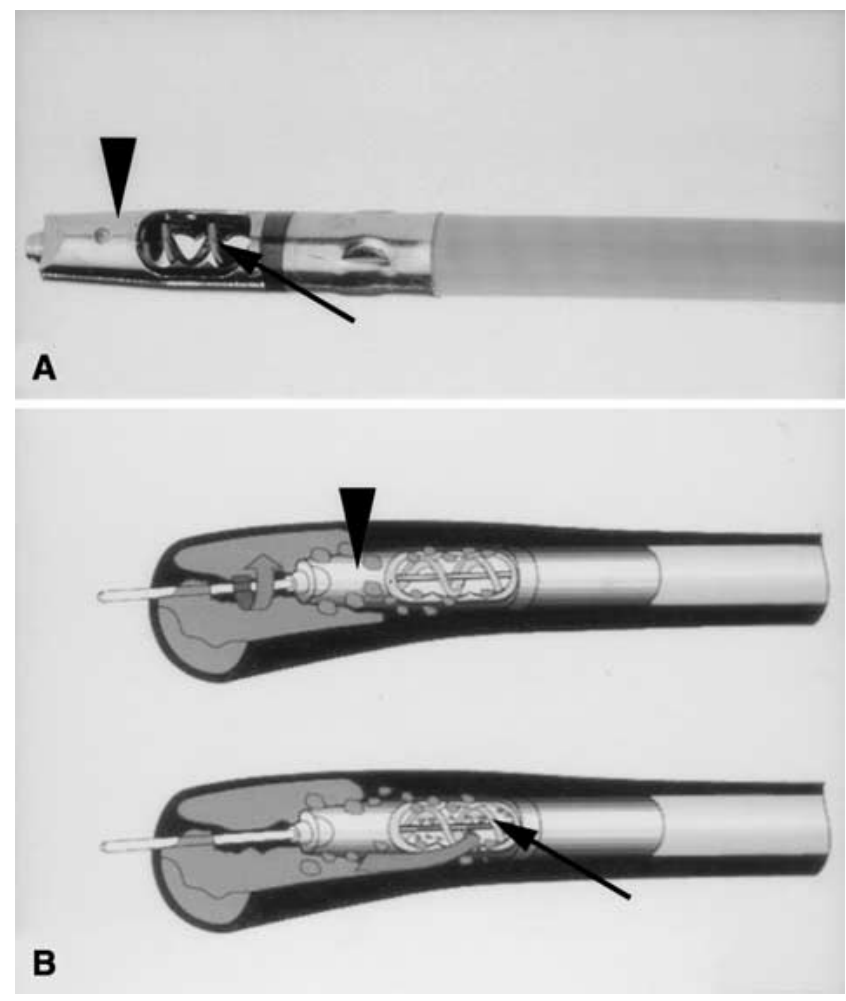

Fig. 1. (A) Distal end/head of the Rotarex mechanical thrombectomy device. (B) Schematic drawing showing the functioning of the head of the Rotarex catheter. The fenestrated rotating dual blade (arrowhead in A and B) produces cutting/grinding and aspiration of the clot material. The motor-driven coated spiral shaft $(40,000 \mathrm{rpm}$; thin arrow in A and $\mathbf{B})$ running through the catheter causes suction $(-435$ $\mathrm{mmHg}$ ) and removal of clot material.

out all wall-adherent thrombotic material. If residual underlying stenoses of $>25 \%$ persist we use additional balloon angioplasty and/or endovascular stents as needed (Fig. 2). In cases of peripheral embolization percutaneous catheter aspiration is our method of choice.

As antithrombotic regimen we use 5000 IU of heparin intraoperatively, followed by low-molecular-weight heparin therapy for 3-7 days and $100 \mathrm{mg}$ of acetylsalicylic acid daily as long-term treatment.

The catheter used in this study was designed for an antegrade approach only. A new design for crossover application has been introduced recently.

\section{Patients}

Forty-one limbs in 38 patients (17 men, 21 women; mean age $75.6 \pm 8.8$ years, range 56-90 years) with occlusion of the femoropopliteal axis were treated with mechanical thrombectomy using the Rotarex system. The ischemic symptomatology was graded according to the Fontaine classification; Rutherford categories are given in parentheses. One patient (2\%) was Fontaine stage IIa (Rutherford 1), 22 patients (54\%) were stage IIb (Rutherford 2-3), 14 patients (34\%) were stage III (Rutherford 4), and 4 patients (10\%) were stage IV (Rutherford 5-6). 

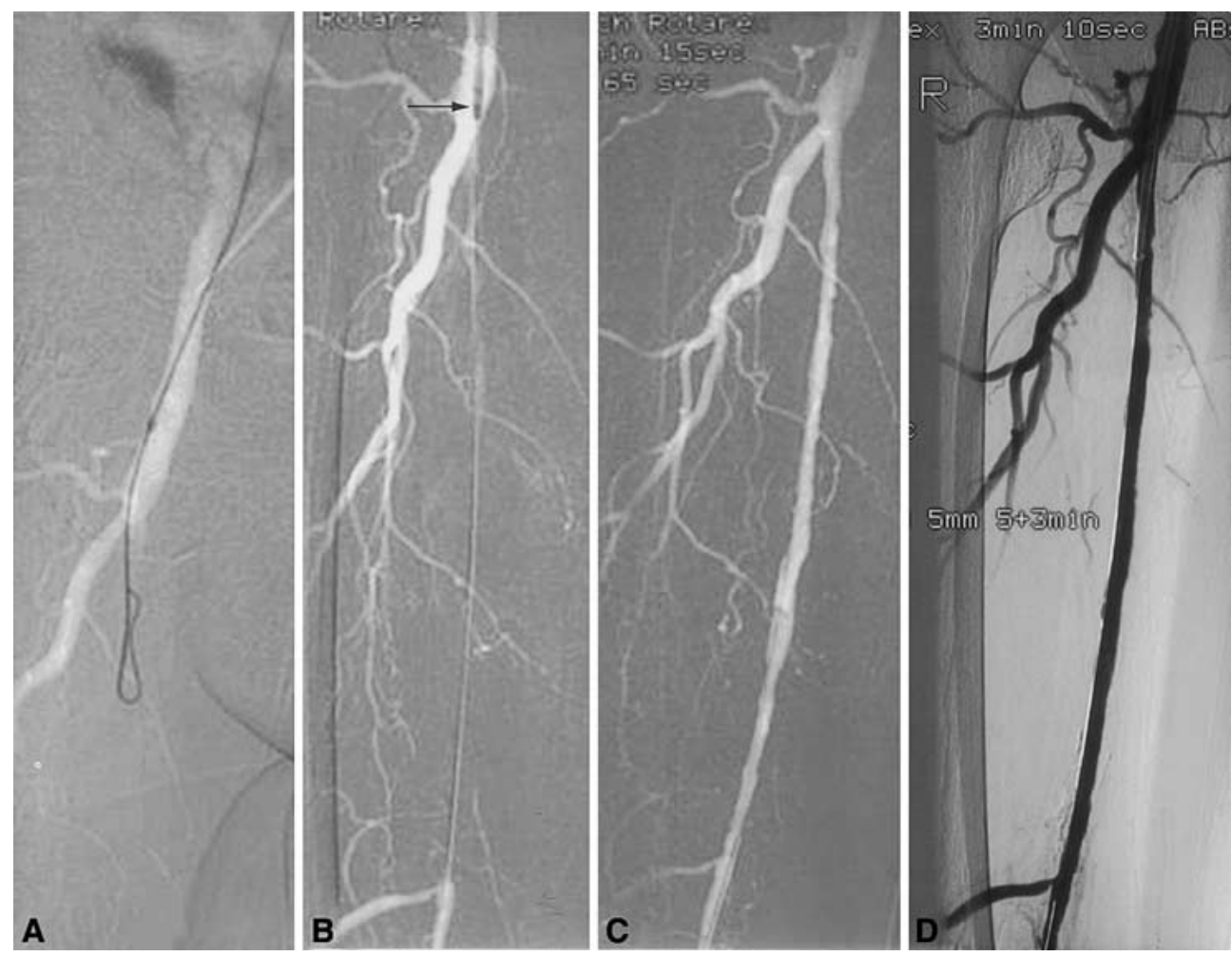

Fig. 2A-D. An 84-year-old patient with Fontaine stage III symptomatology of the right leg for 20 days. (A) Digital subtraction angiography (DSA) shows an occlusion of the superficial femoral artery (SFA). (B) DSA shows the guidewire crossing the $20 \mathrm{~cm}$ long occlusion. Note the head of the Rotarex catheter (arrow) in the common femoral artery, directly before the occlusion. (C) DSA after two passages with the Rotarex catheter (135 sec and $65 \mathrm{sec}$ ) shows the recanalized segment of the SFA. (D) Angiography after additional PTA.

The age of the vascular occlusions varied between 1 and 180 days with an average of $31.6 \pm 33.8$ days. The stages of occlusion were defined as follows: acute occlusion: $\leq 14$ days old, subacute occlusion 15-90 days old, and chronic occlusion more than 90 days old. There were 16 acute occlusions (mean $8 \pm 4.8$ days, range 1-14 days), 24 subacute occlusions (mean $41.1 \pm 21$ days, range 16-89 days), and 1 patient with a chronic occlusion 180 days old. In 2 cases the duration of the occlusion could not be precisely dated but was more than 2 weeks according to the clinical history. These 2 patients have been included in the pool of the subacute lesions but have been disregarded for the calculation of the mean lesion age. The occlusion's length varied between 2 and $35 \mathrm{~cm}$, with a mean of $13.1 \pm 7.5$ $\mathrm{cm}$. Nineteen occlusions were $\leq 10 \mathrm{~cm}, 22$ were $>10 \mathrm{~cm}$. The localization of the occlusions along the femoropopliteal axis was as follows: 26 occlusions were located in the proximal half of the superficial femoral artery (SFA), 15 occlusions were located in the distal half of the SFA including the proximal half of the popliteal artery. There were no isolated occlusions of the popliteal artery.

The inclusion criteria were: (1) an occlusion situated in the femoropopliteal axis with a patent distal popliteal artery and trifurcation; (2) successful passage of the occlusion with a guidewire followed by Rotarex thrombectomy as the primary form of treatment. Exclusion criteria were an occlusion of the distal popliteal artery and trifurcation, the known presence of a popliteal aneurysm, coagulopathy or a blood creatinine value of $\geq 300 \mu \mathrm{g} / \mathrm{l}$, and subintimal recanalization with the guidewire.

The pre-interventional evaluation consisted of the clinical status, including an oscillographic examination at three levels (thigh, leg, and great toe) and brachial-ankle index (BAI), as well as a color duplex examination to evaluate the extent of the occlusion, and the patency of the distal popliteal and tibial arteries.

The same protocol was used for follow-up at $48 \mathrm{hr}, 3,6$, and 12 months after the intervention. The threshold of significant reste- nosis was set at $>50 \%$ as estimated on color Doppler flow measurements [13].

\section{Statistical Analysis}

Quantitative data are expressed as mean values \pm 1 standard deviation. The Kaplan-Meier method was used to calculate the cumulative patency rates. The Wilcoxon rank signed test was used to evaluate intraindividual differences of a variable before and after treatment. The log rank test was used to compare Kaplan-Meier survival curves. Statistical analysis was done with the SPSS statistical software (SSPS 12, Chicago, IL).

\section{Definitions}

Technical success was defined angiographically in the presence of a final residual luminal stenosis of less than $25 \%$. If this goal was not reached with Rotarex treatment only, additional percutaneous transluminal angioplasty (PTA) and/or stent-graft placement was performed in the same session.

Primary patency was defined as an uninterrupted patency with no procedures performed on or at the margins of the treated segment [14]. Secondary patency was defined as restoration of patency by means of any procedure after thrombosis or restenosis [14].

\section{Results}

\section{Technical Success}

All treated limbs were recanalized with a markedly improved lumen after an average of two passages (one to four) 
Table 1. Complications during or following recanalization with the Rotarex device

\begin{tabular}{lll}
\hline & $\begin{array}{l}\text { Major } \\
\text { complications }\end{array}$ & $\begin{array}{l}\text { Minor } \\
\text { complications }\end{array}$ \\
\hline $\begin{array}{l}\text { Arteriovenous fistula } \\
\text { Significant hematoma }\end{array}$ & 1 & \\
$\begin{array}{l}\text { Vessel perforation } \\
\text { Peripheral embolization }\end{array}$ & 1 & 4 \\
$\begin{array}{l}\text { Pseudoaneurysm following } \\
\quad \text { vessel perforation }\end{array}$ & 10 \\
Pseudoaneurysm at puncture site & 1 & 4 \\
\hline
\end{tabular}

Numbers indicate the number of patients.

with the Rotarex device. The mean duration per passage was $42 \pm 31 \mathrm{sec}$ (range 10-180 sec). During the intervention, an average of $122 \pm 50.5 \mathrm{ml}$ (range $50-200 \mathrm{ml}$ ) blood with debris was aspirated into the collecting bag. All but 2 of 41 limbs $(95 \%)$ had a residual stenosis of $>25 \%$. These patients underwent additional PTA in the same session. Three patients were stented (Jostent, Jomed, Beringen, Switzerland; Peiron Stent, Biotronic Bülach, Switzerland) for a dissection after PTA, and one additional case was stented in order to treat a stenosis distal to the recanalized segment. In one case a Wallstent (Boston Scientific, Galway, Ireland) was placed for a vessel perforation with a persistent leak after unsuccessful prolonged balloon inflation. Two limbs were free of stenosis after the passage of the Rotarex device alone, and had no additional PTA. Thus a primary technical success of $100 \%$ was achieved.

\section{Complications}

The complications are listed in Table 1. Of the $21(51 \%)$ complications encountered only $2(5 \%)$ were major. One patient developed a large hematoma at the puncture site, which required transfusion of 2 units of blood. In a second patient an arteriovenous fistula at the level of the recanalized segment spontaneously thrombosed after unsuccessful primary prolonged balloon dilatation. The other complications were rated as minor following the criteria of the SCVIR [14]. Three arterial perforations were successfully sealed with prolonged balloon dilatation (5-10 min) (Fig. 3). One of these showed formation of a pseudoaneurysm at the site of previous perforation at the 3 months follow-up examination; after placement of a stent-graft (Jostent, Jomed, Beringen, Switzerland) successful occlusion of the perforation was obtained. One perforation was treated with placement a Wallstent (Boston Scientific, Galway, Ireland) after unsuccessful prolonged balloon inflation. The color duplex examination at $48 \mathrm{hr}$ showed no vascular leak through the meshes of the stent. In 10 of 41 (24\%) thrombectomy procedures peripheral embolizations were noted: 5 after Rotarex thrombectomy itself, and 5 after additional PTA. Six that were clinically relevant were treated by aspiration. Two of these required additional thrombolysis with $80,000 \mathrm{IU}$ and $100,000 \mathrm{IU}$ of urokinase, respectively. In one of these latter 2 patients an initially unrecognized popliteal aneurysm had been recanalized. Four clinically irrelevant embolisms were left untreated. Four pseudoaneurysms detected at duplex ultrasound after 2 days at the puncture site were treated conservatively by ultrasound-guided compression [2] or thrombosed spontaneously [2].

\section{Clinical Success and Follow-up}

Two patients presented with an early reocclusion within 24 hr. One patient was recanalized two times for acute reocclusion with aspiration and PTA. Repeated treatment with the Rotarex catheter was not possible due to the occlusion of the entire SFA which precluded safe antegrade access of the device. Because of subsequent reocclusion, a surgical bypass was performed 6 weeks later. The second patient showed an early reocclusion 1 day following the initial successful thrombectomy. The spontaneous amelioration of the clinical symptoms rendered a surgical bypass unnecessary. One patient with early reocclusion at 8 and 13 days was successfully treated with thrombolysis and catheter aspiration. Due to the oral anticoagulation of the patient and the formation of a pseudoaneurysm with a local hematoma at the puncture site following the Rotarex procedure, the use of a large $8 \mathrm{Fr}$ introducer sheath had to be avoided. A further patient with a stent in the SFA occluded 15 days after the first Rotarex procedure. Repeat thrombectomy with the Rotarex device, completed by catheter aspiration, ended in a good final result. One patient benefited from an iliofemoral surgical bypass 8 months after the recanalization for an acute occlusion of the external iliac artery; the previously recanalized segment (SFA) was still open at the time of surgery.

At the 3 months follow-up control the rate of significant restenosis was $23 \%(7 / 30)$, at the 6 months control it was $48 \%(13 / 27)$, and at 12 months control it was 39\%(10/26). Only 2 of all recurrences were complete occlusions. The first was detected at the 6 months control and required a surgical bypass operation. The second occlusion was diagnosed at the 12 months control and was successfully treated with repeat thrombectomy with the Rotarex device.

The mean follow-up was 12 months (range 1 day to 51 months). The primary cumulative patency rate was $87 \%$ at 3 months, $62 \%$ at 6 months, and $39 \%$ at 12 months (Fig. 4). The secondary patency rate was $95 \%$ at 3 months, $84 \%$ at 6 months, and $68 \%$ at 12 months (Fig. 5). No statistically significant difference was found between the primary and secondary patency rates of the entire patient pool compared with subgroups defined according to occlusion length, occlusion localization, and occlusion age.

The BAI, including in cases with reobstruction, was significantly $(p<0.01)$ improved after thrombectomy, rising from 0.41 before treatment to 0.92 immediately after treatment and remaining at relatively steady values of 0.93 at 3 months, 0.92 at 6 months, and 0.9 at 12 months. 

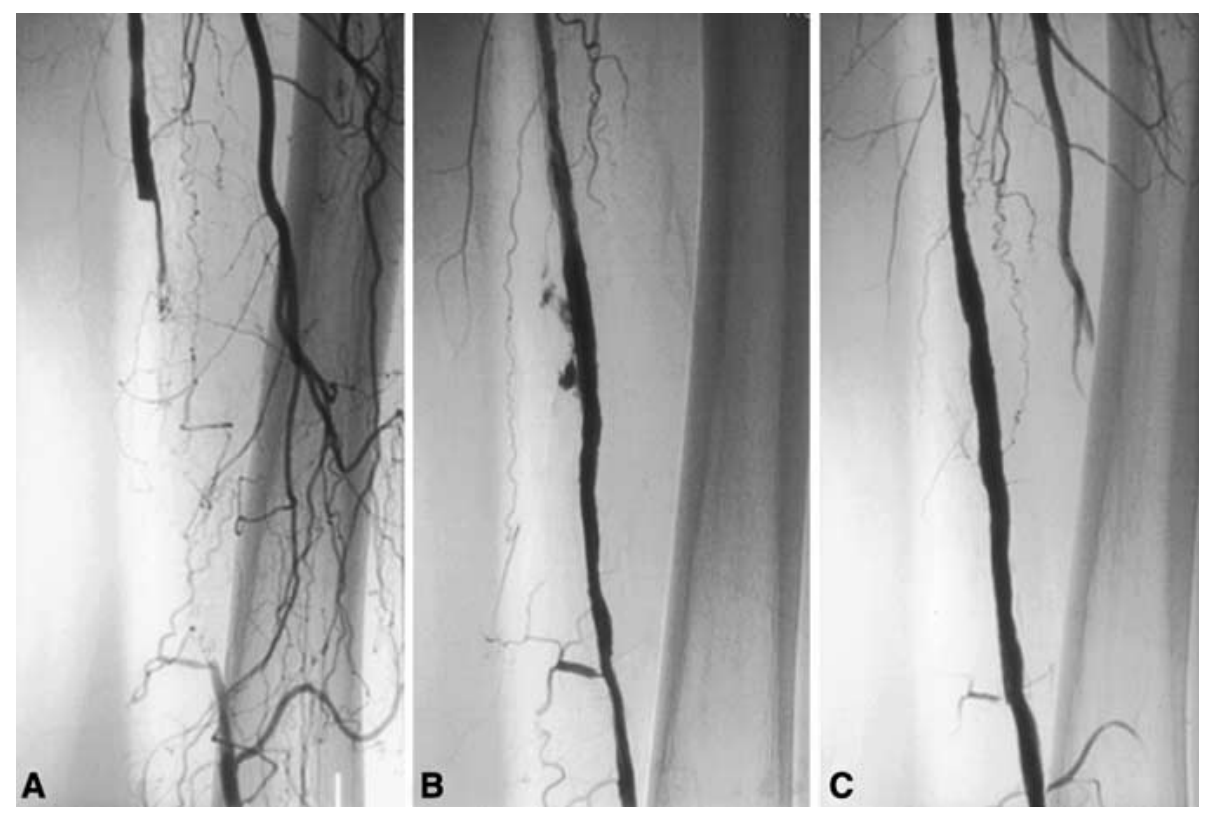

Fig. 3A-C. A 61-year-old patient presenting with Fontaine stage Ilb ischemic symptomatology for 25 days. (A) Angiography shows a 10 $\mathrm{cm}$ long occlusion of the SFA. (B) After two passages with the Rotarex thrombectomy device a vessel perforation is noted at the level of the former occlusion. (C) Angiography after prolonged dilatation (two times $5 \mathrm{~min}$ ) shows sealing of the perforation with a widely patent lumen of the SFA.

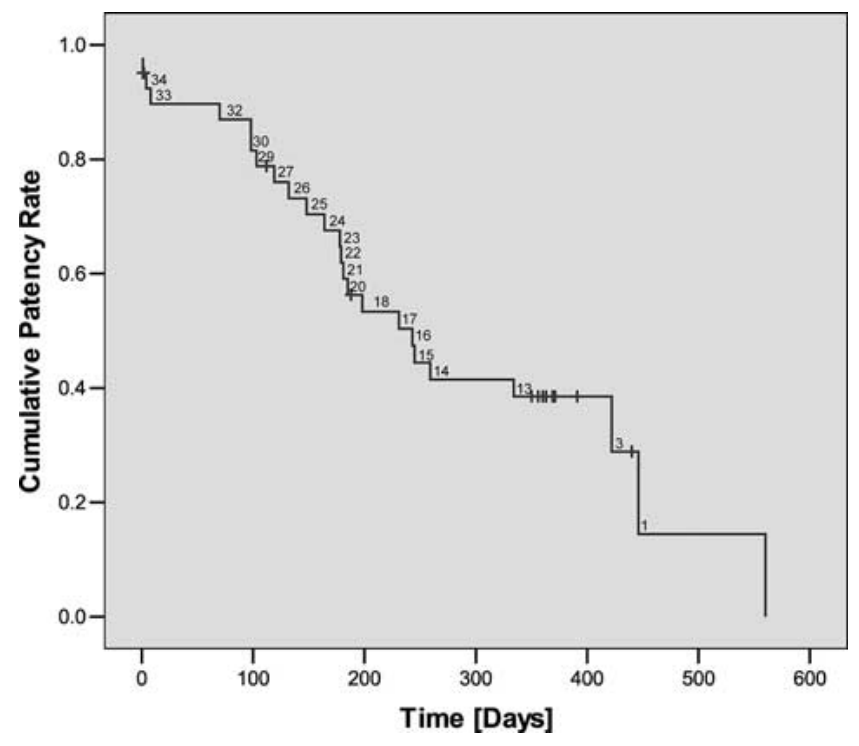

Fig. 4. Primary patency rate of 41 femoropopliteal arteries after recanalization with the Rotarex mechanical thrombectomy device.

Clinically, the Fontaine stages showed improvement of the clinical symptoms. Before thrombectomy the majority of patients (98\%) were Fontaine stage IIb or above. At the 3 months control $97 \%$ of the patients were either stage I (23) or IIa (6). This rate decreased slowly at 6 months (81\%) and 12 months (85\%) (Fig. 6). Among the patients who had a significant restenosis at follow-up the majority were either asymptomatic or only slightly symptomatic, with $86 \%, 73 \%$, and $75 \%$ being Fontaine stages I-IIa at 3, 6 , and 12 months, respectively. All the patients without significant restenosis were Fontaine stage I-IIa at 3 and 12 months, while only 1 patient of $10(10 \%)$ was stage $\mathrm{IIb}$ at 6 months.

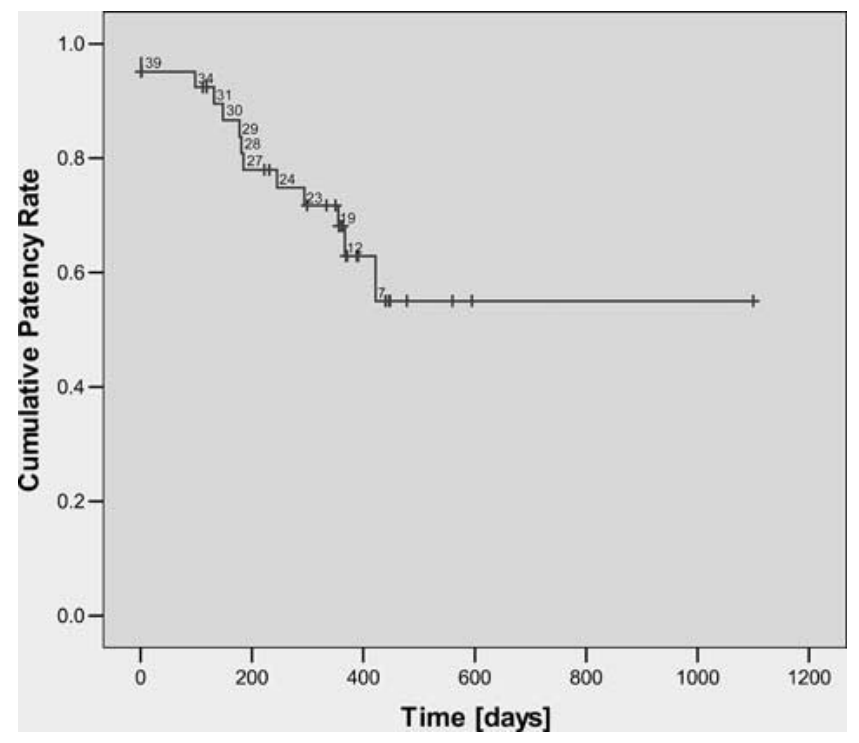

Fig. 5. Secondary patency rate of 41 femoropopliteal arteries after recanalization with the Rotarex mechanical thrombectomy device.

None of our patients underwent amputation in the 12 months follow-up. Only one patient needed a surgical bypass 6 weeks after the thrombectomy procedure due to repeated early reocclusions.

During the 12 months follow-up 6 patients died. The mean age of these patients was $79 \pm 12$ years. Their death was neither directly nor indirectly related to the thrombectomy procedure.

\section{Discussion}

Percutaneous catheter aspiration thrombo-embolectomy is the simplest and most cost-effective mechanical way to treat 
Fontaine Staging

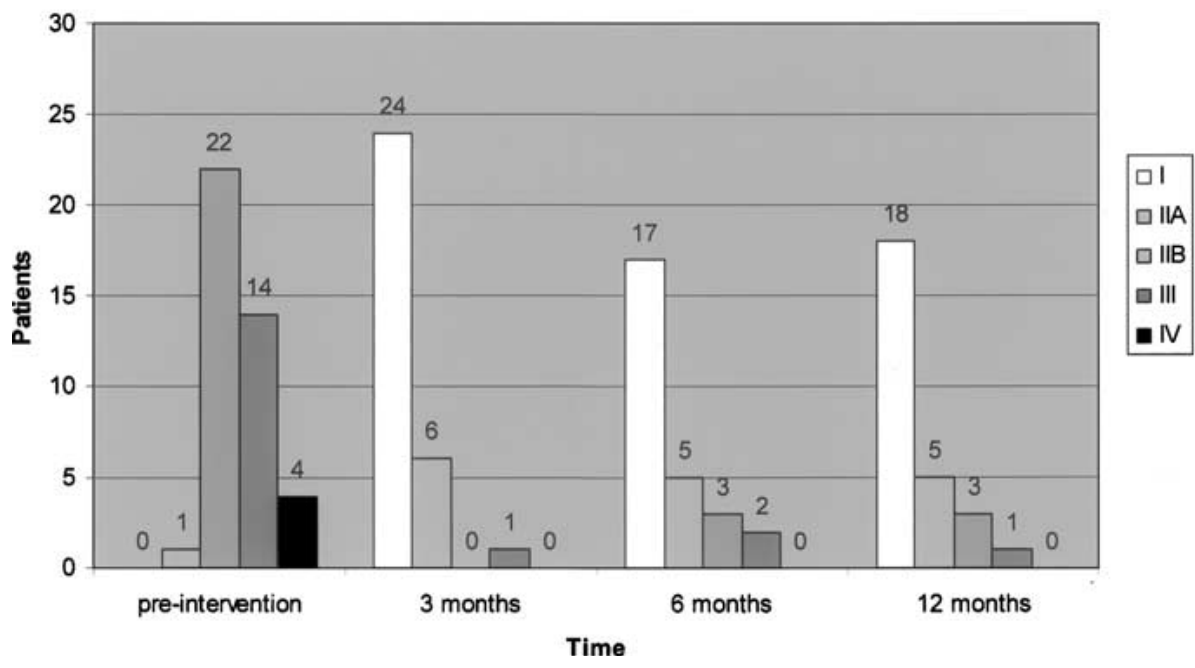

Fig. 6. Evolution of the Fontaine stage for clinical classification of limb ischemia before, and 3,6, and 12 months after femoropopliteal recanalization. acute femoropopliteal occlusions. Acute popliteal emboli in particular may be treated without any additional thrombolysis or even the need for balloon dilatation if there is no underlying or concomitant atherosclerotic lesion [2, 15]. However, since in native arteries there seems to be rapid onset of thrombus organization thrombi start to become adherent to the vascular wall within a couple of days, rendering catheter aspiration without additional lysis difficult or insufficient. In addition many occlusions are caused by an underlying plaque and/or thrombus, so the ideal thrombectomy device should also be capable of removing organized thrombus $[3,16]$. Data for MT using rotational recirculation devices which work by the vortex principle or hydrodynamic (rheolytic) recirculation devices report success rates in the lower extremity arteries of 66-90\% [1, 7-9, 17-19]; however, adjunctive thrombolysis (usually overnight) was required in $20-42 \%$ of cases $[1,7-9,17,18]$. Rousseau et al. reported success for MT alone in $61 \%$ of their cases, which was increased to $83 \%$ by adding thrombolytic therapy [1, 17]. Morgan and Belli [1] concluded that mechanical thrombectomy devices are useful for the treatment of thrombosed dialysis grafts but less effective in native arteries and bypass grafts.

The Rotarex catheter was especially designed to address removal of organized, solid thrombus, while the possibility of plaque removal cannot be excluded [11]. Its construction is relatively simple, with a rotating windowed cutting head and only a single lumen for a coaxial guidewire and a spiral which transports the aspirated material through the catheter into a collecting bag. The unique feature of this device consists of a radial expansion coefficient of 2 in fresh and partly organized thrombus, which means that a diameter of $2.6 \mathrm{~mm}(8 \mathrm{Fr})$ clears a lumen of more than $5 \mathrm{~mm}$ [12]. It is feasible to use this device in arteries with a diameter of 4-7 mm [11, 20]. To use the device once the occlusion has been crossed with a guidewire is easy.
The technical and initial clinical results demonstrate equal success in long and short acute and subacute occlusions of the femoropopliteal artery. Therefore the device seems very useful in clinical practice in cases where a long procedure is not easily tolerated by the patient or in cases where the use of thrombolysis is contraindicated or carries an increases risk of bleeding complications.

In our study, the technical success rate was $100 \%$ (41/41). To optimize the immediate result after thrombectomy with the Rotarex catheter ( $>25 \%$ residual lumen reduction, mostly due to underlying chronic plaque formation) PTA was performed in $95 \%$ of cases, with additional stenting in 3 limbs because of a local dissection following PTA and for a recoiling lesion distal to the initial obstruction in another case. Furthermore another patient benefited from stent placement after unsuccessful prolonged balloon inflation of an arterial perforation. Additional thrombolysis was needed in only 2 patients $(5 \%)$ in whom peripheral emboli after thrombectomy could not be fully aspirated by PTA. Thrombolysis was never used as an adjunctive measure for the primary recanalization procedure. Using the same device Bérczi et al. [21] and Zeller et al. [20] reported similar primary success rates of $94 \%$ and $96 \%$, respectively. Only in 2 patients was additional local thrombolysis used to treat symptomatic perinterventional peripheral emboli [20], while the rates for additional thrombolysis in mechanical thrombectomy reported in the literature vary between $58 \%$ and $100 \%$ [1, 3, 4, 7, 16, 20-23]. Mechanical maceration $[16,20,21]$ seems to give slightly better results than hydrodynamic approaches [1, 3, 7, 22]. In some studies PTA also showed high success rates in the range of $90-95 \%$ [1-4, 24]. However, additional thrombolysis was used in $71 \%$ [24] and 22\% [4] of cases, respectively. In the report by Zehnder et al. [4] $87.5 \%$ of patients had acute onset of occlusion less than 14 days old and no patient had an occlusive episode of more than 4 weeks. In addition the 
history of acute symptoms in the patients who did not need thrombolysis was only approximately 5 days. It should be mentioned that many of the emboli treated with aspiration were either due to PTA or thrombus residue after thrombolysis [10] After prolonged thrombolysis recanalization rates vary between $79.7 \%$ and $85 \%$ for acute or subacute occlusions [25, 26].

Periprocedural peripheral embolism occurred in $24 \%$ of our cases (10/41), five times during thrombectomy with the Rotarex and five times after subsequent dilatation, but no clinically relevant sequelae occurred after additional catheter aspiration or lysis. Zeller et al. [20] reported a much lower rate $(10 \%)$ of peripheral embolization and never during the application of the Rotarex, but only after additional angioplasty. Berczi et al. [21] had a cohort of patients similar to ours, as regards age as well as Fontaine stages, but also reported a lower rate of peripheral embolism (18\%). In 2 of the 3 cases, emboli occurred during recanalization with the Rotarex catheter and all were treated with additional aspiration. However, according to our own learning curve peripheral embolization can be markedly reduced by slow advancement and multiple retractions of the catheter back to the unoccluded segment in order to clean out as much loosened material as possible before reestablishing flow by crossing the most distal part of the occlusion. The rate of peripheral embolization is similar in other reports of mechanical thrombectomy, ranging usually between $3 \%$ and $10 \%$, but may be as low as 0 and as high as $28 \%[1,22]$. In many of these reports a high proportion of patients were routinely treated with additional local thrombolysis [22].

The complication rate $(51 \%)$ in our study may appear rather high, but apart from one large groin hematoma and an arteriovenous fistula at the level of the recanalized segment the complications were mostly of minor or no consequence and could be treated by means of endovascular techniques during the same procedure, such as aspiration of peripheral emboli. None needed surgical treatment or amputation. Four small pseudoaneurysms were picked up at the 1-2 days duplex ultrasound control which otherwise might have not been detected at all because of spontaneous closure. In our experience the relatively large size of the catheter sheath $(8$ Fr) was not coupled with an increased risk of bleeding and pseudoaneurysm formation at the puncture site compared with alternative techniques such as catheter aspiration, which also needs introducer sheaths of at least 8 Fr for the femoropopliteal axis.

A potential complication which seems unique to the Rotarex device is vessel wall perforation, which may even lead to an arteriovenous fistula. We encountered four perforations, one pseudoaneurysm formation at the site of previous perforation at the 3 months follow-up examination and one perforation with a concurrent arteriovenous fistula. This complication has also been reported in 2 of 19 treated limbs by Bérczi et al. [21], and 3 of 31 (10\%) femoropopliteal occlusions in the study by Zeller et al. [20] and were treated in a similar fashion. As in our patients, these patients had heavily calcified vessels. Presumably the rather sharp edges of the rotating catheter tip may catch at a plaque, thereby causing rupture of the vessel wall. Therefore care should be taken and patients with significant calcifications at the site of occlusion may be better not treated with this device. To avoid embolizations a slow advancement and repeated retraction of the catheter proximal to the occlusion before crossing the most distal part of the occlusion is essential.

In summary, apart from the above-mentioned vessel wall perforation the complications are not specifically related to this device and no different from those of other instruments, though the incidence of hemorrhagic complications definitely seems lower than in techniques using additional thrombolysis [3, 22, 25, 27].

The restenosis rates in our study are similar to those published by Zeller et al. [16, 20], with rates of $33 \%$ and $37 \%$ at 6 and 12 months, respectively. Studies concerning thrombolytic therapy, notably the STILE study [2], also noted significant restenosis rates at 1 year, varying between $32 \%$ and $36 \%$. The primary and secondary patency rates tally with the data of Berczi et al. [21]. These authors describe primary patency rates of $39 \pm 13 \%$ at 6 and 12 months with steady patency rates of about $40 \%$ during 19 months follow-up and secondary patency rates of $53 \pm 13 \%$. In our experience the long-term primary patency was $21 \%$ at 15 months and $10 \%$ at 18.5 months. These results have to be interpreted with caution due to the very small number of patients .

In our hands the costs involved using the Rotarex catheter are approximately one fifth higher than for thrombolysis and catheter aspiration (1000 Euro vs. 800 Euro). Although we did not record procedure time, from personal experience using alternative techniques we feel that the Rotarex procedure saves a considerable amount of time and therefore is cost-effective.

In conclusion, the Rotarex mechanical thrombectomy device is an efficient, quick, easy to handle, and safe tool for the treatment of acute, subacute or even chronic peripheral arterial thromboembolic occlusions. It can be used for short or long occlusions with equal success provided the obstruction is not heavily calcified and has been safely passed with a guidewire first.

\section{References}

1. Morgan R, Belli AM (2002) Percutaneous thrombectomy: A review. Eur Radiol 12:205-217

2. Wagner HJ, Starck EE (1992) Acute embolic occlusions of the infrainguinal arteries: Percutaneous aspiration embolectomy in 102 patients. Radiology 182:403-407

3. Sharafuddin MJ, Hicks ME (1998) Current status of percutaneous mechanical thrombectomy. III. Present and future applications. J Vasc Interv Radiol 9:209-224

4. Zehnder T, Birrer M, Do DD, et al. (2000) Percutaneous catheter thrombus aspiration for acute or subacute arterial occlusion of the legs: How much thrombolysis is needed? Eur J Vasc Endovasc Surg 20:41-46 
5. Sharafuddin MJ, Hicks ME (1997) Current status of percutaneous mechanical thrombectomy. I. General principles. J Vasc Interv Radiol 8:911-921

6. Sharafuddin MJ, Hicks ME (1998) Current status of percutaneous mechanical thrombectomy. II. Devices and mechanisms of action. J Vasc Interv Radiol 9:15-31

7. Rilinger N, Gorich J, Scharrer-Pamler R, et al. (1997) Short-term results with use of the Amplatz thrombectomy device in the treatment of acute lower limb occlusions. J Vasc Interv Radiol 8:343-348

8. Reekers JA, Kromhout JG, Spithoven HG, et al. (1996) Arterial thrombosis below the inguinal ligament: Percutaneous treatment with a thrombosuction catheter. Radiology 198:49-53

9. Tadavarthy SM, Murray PD, Inampudi S, et al. (1994) Mechanical thrombectomy with the Amplatz device: Human experience. J Vasc Interv Radiol 5:715-724

10. Drasler WJ, Jenson ML, Wilson GJ, et al. (1992) Rheolytic catheter for percutaneous removal of thrombus. Radiology 182:263-267

11. Schmitt HE, Jager KA, Jacob AL, Mohr H, Labs KH, Steinbrich W (1999) A new rotational thrombectomy catheter: System design and first clinical experiences. Cardiovasc Intervent Radiol 22:504-509

12. Zana K, Otal P, Fornet B, et al. (2001) In vitro evaluation of a new rotational thrombectomy device: The Straub Rotarex catheter. Cardiovase Intervent Radiol 24:319-323

13. Ranke C, Rieder M, Creutzig A, et al. (1995) A nomogram of duplex ultrasound quantification of peripheral arterial stenoses. Studies of the cardiovascular model and in angiography patients. Med Klin (Munich) 90:72-77

14. Sacks D, Marinelli DL, Martin LG, et al. (1997) Reporting standards for clinical evaluation of new peripheral arterial revascularization devices. Technology Assessment Committee. J Vasc Interv Radiol 8:137149

15. Starck EE, McDermott JC, Crummy AB, et al. (1985) Percutaneous aspiration thromboembolectomy. Radiology 156:61-66

16. Zeller T, Muller C, Frank U, et al. (2001) [The Straub-Rotarex thrombectomy system: initial experiences]. Rofo Fortschr Geb Rontgenstr Neuen Bildgeb Verfahr 173:626-631
17. Rousseau H, Sapoval M, Ballini P, et al. (1997) Percutaneous recanalization of acutely thrombosed vessels by hydrodynamic thrombectomy (Hydrolyser). Eur Radiol 7:935-941

18. Wagner HJ, Muller-Hulsbeck S, Pitton MB, et al. (1997) Rapid thrombectomy with a hydrodynamic catheter: Results from a prospective, multicenter trial. Radiology 205:675-681

19. Henry M, Amor M, Henry I, et al. (1998) The Hydrolyser thrombectomy catheter: A single-center experience. J Endovasc Surg 5:2431

20. Zeller T, Frank U, Burgelin K, et al. (2002) [Long-term results after recanalization of acute and subacute thrombotic occlusions of the infraaortic arteries and bypass-grafts using a rotational thrombectomy device]. Rofo Fortschr Geb Rontgenstr Neuen Bildgeb Verfahr 174:1559-1565

21. Berczi V, Deutschmann HA, Schedlbauer P, et al. (2002) Early experience and midterm follow-up results with a new, rotational thrombectomy catheter. Cardiovasc Intervent Radiol 25:275-281

22. Kasirajan K, Haskal ZJ, Ouriel K (2001) The use of mechanical thrombectomy devices in the management of acute peripheral arterial occlusive disease. J Vasc Interv Radiol 12:405-411

23. Kasirajan K, Gray B, Beavers FP, et al. (2001) Rheolytic thrombectomy in the management of acute and subacute limb-threatening ischemia. J Vasc Interv Radiol 12:413-421

24. Wagner HJ, Starck EE, Reuter P (1994) Long-term results of percutaneous aspiration embolectomy. Cardiovasc Intervent Radiol 17:241246

25. Ouriel K, Veith FJ, Sasahara AA (1998) A comparison of recombinant urokinase with vascular surgery as initial treatment for acute arterial occlusion of the legs. Thrombolysis or Peripheral Arterial Surgery (TOPAS) Investigators. N Engl J Med 338:1105-1111

26. Jung EM, Lutz R, Rupp N (2000) [Low-dose thrombolysis using rt-Pa in extensive peripheral vascular occlusion]. Rofo Fortschr Geb Rontgenstr Neuen Bildgeb Verfahr 172:1028-1034

27. Kasirajan K, Ouriel K (2000) Management of acute lower extremity ischemia: Treatment strategies and outcome. Curr Interv Cardiol Rep 2:119-129 